\title{
Topological entropy for locally linearly compact vector spaces and field extensions
}

https://doi.org/10.1515/taa-2020-0005

Received December 31, 2018; accepted January 31, 2020

Abstract: Let $\mathbb{K}$ be a discrete field and $(V, \phi)$ a pair consisting of a locally linearly compact $\mathbb{K}$-space $V$ and a continuous endomorphism $\phi: V \rightarrow V$. We provide the formulae to compute the topological entropy ent ${ }^{\star}$ of the flow $(V, \phi)$ subject to either extension or restriction of scalars.

Keywords: linearly compact vector space, locally linearly compact vector space, topological entropy, continuous endomorphism, extension, restriction, algebraic dynamical system.

2010 MSC: 37A35; 15A03; 15A04; 20K30; 22B05.

\section{Introduction}

An entropy function over a category $\mathcal{C}$ can be regarded as an invariant

$$
\mathrm{h}: \operatorname{Flow}(\mathcal{C}) \rightarrow \mathbb{N} \cup\{\infty\},
$$

of the category Flow(C), whose objects are the flows defined over $\mathcal{C}$. Recall that a flow over an arbitrary category $\mathcal{C}$ is a pair $(C, \gamma)$ consisting of an object $C$ and an endomorphism $\gamma: C \rightarrow C$.

The first appearance of topological entropy was in 1965 when Adler, Konheim and McAndrew [1] defined it for continuous self-maps of compact spaces. Afterwards, Bowen [3] and Hood [7] brought a notion of topological entropy into the world of uniformly continuous self-maps on uniform spaces (also known as uniform entropy) opening up the possibility for computing entropy of any given continuous endomorphism $\phi: G \rightarrow G$ of a topological group $G$. E.g., profinite groups and, more generally, locally compact groups.

Interest in the locally compact case is currently very high because locally compact groups are omnipresent in several areas of mathematics. In particular, when the locally compact group is also totally disconnected, the topological entropy becomes more docile leading to various significant results. For example, for totally disconnected locally compact groups and their endomorphisms, there exists a strict relation between the topological entropy $h_{\text {top }}$ and the famous scale function $s$ defined in [10] (see [2, 8] for details).

By analogy with the topological entropy $h_{\text {top }}$, the topological entropy ent ${ }^{\star}$ has been introduced in [6] for the category $\mathbb{K}_{\mathbb{L}} L L C$ of locally linearly compact vector spaces over a discrete field $\mathbb{K}$, and the main motivation for studying such an entropy function was to reach a better understanding of $h_{t o p}$ by means of the more rigid case of locally linearly compact vector spaces. A topological vector space $V$ over a discrete field $\mathbb{K}$ is said to be locally linearly compact if $V$ admits a local basis at 0 consisting of linearly compact open $\mathbb{K}$-subspaces. Namely, every neighbourhood of 0 contains an open $\mathbb{K}$-subspace that is linearly compact in the subspace topology. Recall that a topological vector space $E$ is linearly compact when

(LC1) it is a Hausdorff space in which there is a local base of 0 consisting of linear subspaces of $E$;

(LC2) any filter base on $E$ consisting of closed linear varieties has a non-empty intersection.

^Corresponding Author: Ilaria Castellano: University of Udine, Viale delle Scienze 206, Udine 33100 (ITALY), E-mail: ilaria.castellano88@gmail.com 
Notice that, for a finite field $\mathbb{K}$, every locally linearly compact $\mathbb{K}$-space $V$ is a totally disconnected locally compact abelian group and

$$
h_{\text {top }}(V, \phi)=\operatorname{ent}^{\star}(V, \phi) \cdot \log |\mathbb{K}|,
$$

for every continuous endomorphism $\phi: V \rightarrow V$ (see [6, Proposition 3.9]). In the latter equation it is clear that the size of the field $\mathbb{K}$ affects - as predictable - the value of the topological entropy. Therefore, it is reasonable to ask whether the topological entropy is affected by the change of scalars also in the case of infinite fields.

The aim of this paper is to address this question, which finds a complete answer in Theorem 4.5. Namely, we first see that both the procedures of restriction and extension of scalars continue to be available in the context of locally linearly compact vector spaces: given a finite field extension $\mathbb{F} \leq \mathbb{K}$, there exist functors

$$
\operatorname{Res}_{\mathbb{F}}^{\mathbb{K}}(-):{ }_{\mathbb{K}} L L C \rightarrow{ }_{\mathbb{F}} L L C \text { and } \quad \operatorname{Ind}_{\mathbb{F}}^{\mathbb{K}}(-):{ }_{\mathbb{F}} L L C \rightarrow{ }_{\mathbb{K}} L L C
$$

forming an adjunction by

$$
\operatorname{CHom}_{\mathbb{K}}\left(\operatorname{Ind}_{\mathbb{F}}^{\mathbb{K}}(-),-\right) \cong \operatorname{CHom}_{\mathbb{F}}\left(-, \operatorname{Res}_{\mathbb{F}}^{\mathbb{K}}(-)\right),
$$

where CHom is understood to be the space of continuous homomorphisms. Afterwards, for finite field extensions $\mathbb{F} \leq \mathbb{K} \leq \mathbb{L}$, we provide the formulae

$$
\left.\operatorname{ent}_{\mathbb{F}}^{\star}\left(\operatorname{Res}_{\mathbb{F}}^{\mathbb{K}}(V, \phi)\right)=[\mathbb{K}: \mathbb{F}] \cdot \operatorname{ent}_{\mathbb{K}}^{\star}(V, \phi) \quad \text { and } \quad \operatorname{ent}_{\mathbb{L}}^{\star}\left(\operatorname{Ind}_{\mathbb{K}}^{\mathbb{L}}(V, \phi)\right)=\operatorname{ent}_{\mathbb{K}}^{\star}(V, \phi), \quad(V, \phi) \in \operatorname{ob}\left(\mathrm{Flow}_{\mathbb{K}} L L C\right)\right) \text {, }
$$

that rule the effect of the change of scalars on the computation of ent ${ }^{\star}$.

There are no surprises in either the statement or the proof of Theorem 4.5, but its content does not appear anywhere else. Indeed this paper is intended to be a complement to $[5,6]$ where ent ${ }^{\star}$ for locally linearly compact $\mathbb{K}$-spaces has been introduced and studied.

\section{Preliminaries and basic properties}

Throughout, the topology on arbitrary fields will always be the discrete topology. We summarise in this section some of the properties of (locally) linearly compact vector spaces and the topological entropy ent ${ }^{\star}$ (see [6, Sections 2 and 3] for references) that will be used in the rest of the paper sometimes with no previous acknowledgement.

A topological $\mathbb{K}$-space is said to be linearly topologized if it admits a local basis at 0 of $\mathbb{K}$-linear subspaces. For linearly topologized $\mathbb{K}$-spaces $V, V^{\prime}$ and $W$ such that $W \leq V$, the following hold:

P1. If $W$ is linearly compact, then $W$ is closed in $V$.

P2. If $V$ is linearly compact and $W$ is closed, then $W$ is linearly compact.

P3. Linear compactness is preserved by continuous homomorphisms.

P4. If $V$ is discrete, then $V$ is linearly compact if and only if $V$ has finite dimension.

P5. If $W$ is closed, then $V$ is linearly compact if and only if $W$ and $V / W$ are linearly compact.

P6. The direct product of linearly compact $\mathbb{K}$-spaces is linearly compact.

P7. An inverse limit of linearly compact $\mathbb{K}$-spaces is linearly compact.

P8. If $V$ is linearly compact, then $V$ is complete.

P9. Let $V$ be a linearly compact $\mathbb{K}$-space. Every continuous $\mathbb{K}$-linear map $f: V \rightarrow V^{\prime}$ is open onto its image.

P10. Linearly compact $\mathbb{K}$-spaces satisfy Lefschetz duality.

P11. Every linearly compact $\mathbb{K}$-space is topologically a direct product of copies of $\mathbb{K}$.

P12. Every linearly compact $\mathbb{K}$-space is topologically an inverse limit of finite-dimensional $\mathbb{K}$-spaces.

\subsection{Complete tensor product and profinite plagiarism}

Given linearly compact $\mathbb{K}$-spaces $V$ and $W$, a linearly compact $\mathbb{K}$-space $T$ (together with a bilinear map $b: V \times$ $W \rightarrow T$ ) is a complete tensor product of $V$ and $W$ over $\mathbb{K}$ if it satisfies the following universal property: for 
every linearly compact $\mathbb{K}$-space $Z$ and every continuous bilinear map $\beta: V \times W \rightarrow Z$ there exists a continuous linear map $\hat{\beta}: T \rightarrow Z$ such that

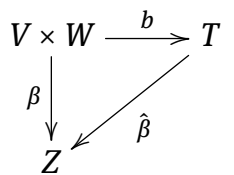

commutes. It is easy to see that if the complete tensor product exists, it is unique up to isomorphism. We denote it by $V \widehat{\otimes}_{\mathbb{K}} W$.

Proposition 2.1. With the above notation, the complete tensor product $V \widehat{\otimes}_{\mathbb{K}} W$ exists. In fact, if

$$
V=\lim _{i \in I} V_{i} \text { and } \quad W=\varlimsup_{\overleftarrow{j \in J}} W_{j},
$$

where each $V_{i}$ (respectively, $\left.W_{j}\right)$ is a finite-dimensional $\mathbb{K}$-space endowed with the discrete topology, then

$$
V \widehat{\otimes}_{\mathbb{K}} W=\lim _{i \in I, j \in J}\left(V_{i} \otimes_{\mathbb{K}} W_{j}\right),
$$

where $V_{i} \otimes_{\mathbb{K}} W_{j}$ is the usual tensor product as abstract $\mathbb{K}$-spaces. In particular, $V \widehat{\otimes}_{\mathbb{K}} W$ is the linearly compactcompletion of $V \otimes_{\mathbb{K}} W$, where $V \otimes_{\mathbb{K}} W$ has the topology for which a fundamental system of neighbourhoods of $O$ are the kernels of the natural maps

$$
V \otimes_{\mathbb{K}} W \rightarrow V_{i} \otimes_{\mathbb{K}} W_{j} \quad(i \in I, j \in J) .
$$

Proof. Basically, we use the strategy developed in the proof of [9, Lemma 5.5.1]. Since

$$
V \times W \cong \text { top } \lim _{i \in I, j \in J}\left(V_{i} \times W_{j}\right),
$$

by the universal property of inverse limits, there exists a canonical continuous $\mathbb{K}$-bilinear map

$$
\iota: V \times W \rightarrow \lim _{i \in I, j \in J}\left(V_{i} \otimes_{\mathbb{K}} W_{j}\right) .
$$

Since every linearly compact $\mathbb{K}$-space is the inverse limit of its finite-dimensional $\mathbb{K}$-quotients, it suffices to check the universal property only for an arbitrary $\mathbb{K}$-space, say $F$, of finite dimension. Suppose $\beta: V \times W \rightarrow F$ to be continuous and $\mathbb{K}$-bilinear. For $\operatorname{dim}_{\mathbb{K}}(F)<\infty$, there is a pair of indices $(i, j) \in I \times J$ and a continuous $\mathbb{K}$-bilinear map $\beta_{i j}: V_{i} \times W j \rightarrow F$ such that the diagram

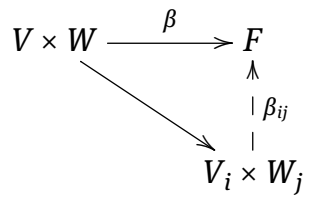

commutes. Now the universal property of $V_{i} \otimes_{\mathbb{K}} W_{j}$ produces a continuous $\mathbb{K}$-bilinear map $\hat{\beta}_{i j}: V_{i} \otimes_{\mathbb{K}} W_{j} \rightarrow F$ such that $\hat{\beta}_{i j}\left(v_{i} \otimes w_{j}\right)=\beta\left(v_{i}, w_{j}\right)$. Finally, define $\beta: V \widehat{\otimes}_{\mathbb{K}} W \rightarrow F$ to be

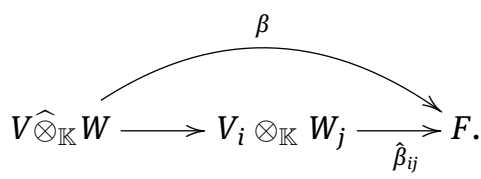


Since the complete tensor product is unique up to isomorphism, the construction above does not depend on the (inverse limit)-representation of $V$ and $W$. Moreover, the right-exact covariant functor $V \widehat{\otimes}_{\mathbb{K}}{ }^{-}:{ }_{\mathbb{K}} \mathrm{LC} \rightarrow{ }_{\mathbb{K}} \mathrm{LC}$ is additive and satisfies

$$
V \widehat{\otimes}_{\mathbb{K}} \mathbb{K} \cong \text { top } V,
$$

where the isomorphism is natural in $V$. Clearly, similar properties hold for $-\widehat{\otimes}_{\mathbb{K}} W$.

Remark 2.2. The tensor products here are merely vector spaces, but complete tensor products can be defined for more general objects, e.g., linearly compact modules over linearly compact commutative rings.

\subsection{Topological entropy on $\mathbb{K}_{\mathbb{K}} L L C$}

Let $(V, \phi)$ be a flow over $\mathbb{K}_{\mathbb{K}} \mathrm{LLC}$ and denote by $\mathcal{B}(V)$ the collection of all linearly compact open $\mathbb{K}$-subspaces of $V$. Then the topological entropy of $(V, \phi)$ is defined to be

$$
\operatorname{ent}_{\mathbb{K}}^{\star}(V, \phi)=\sup _{U \in \mathcal{B}(V)} H_{\mathbb{K}}^{\star}(\phi, U),
$$

where

$$
H_{\mathbb{K}}^{\star}(\phi, U)=\lim _{n \rightarrow \infty} \frac{1}{n} \operatorname{dim}_{\mathbb{K}}\left(U /\left(U \cap \phi^{-1} U \cap \ldots \cap \phi^{-n+1} U\right)\right) .
$$

The linearly compact open $\mathbb{K}$-subspace $U \cap \phi^{-1} U \cap \ldots \cap \phi^{-n+1} U$ is called $n$-cotrajectory of $\phi$ in $U$ and it is denoted by $C_{n}^{\mathbb{K}}(\phi, U)$.

In [6] the topological entropy was denoted simply by ent ${ }^{\star}$ but we need to highlight the field involved.

eP1. (Invariance under conjugation) For every isomorphism $\alpha: V \rightarrow W$ of locally linearly compact $\mathbb{K}$-spaces,

$$
\operatorname{ent}_{\mathbb{K}}^{\star}\left(W, \alpha \phi \alpha^{-1}\right)=\operatorname{ent}_{\mathbb{K}}^{\star}(V, \phi) .
$$

eP2. (Monotonicity) For every closed $\mathbb{K}$-subspace $W$ of $V$ such that $\phi(W) \leq W$,

$$
\operatorname{ent}_{\mathbb{K}}^{\star}(V, \phi) \geq \max \left\{\operatorname{ent}_{\mathbb{K}}^{\star}\left(W, \phi\lceil W), \operatorname{ent}_{\mathbb{K}}^{\star}(V / W, \bar{\phi})\right\}\right.
$$

where $\bar{\phi}: V / W \rightarrow V / W$ is induced by $\phi$.

eP3. (Logarithmic law) For all $k \in \mathbb{N}$, ent $\mathbb{K}_{\mathbb{K}}^{\star}\left(V, \phi^{k}\right)=k \cdot$ ent $_{\mathbb{K}}^{\star}(V, \phi)$.

eP4. (Continuity on inverse limits) Let $\left\{W_{i} \mid i \in I\right\}$ be a directed system (for inverse inclusion) of closed $\mathbb{K}$ subspaces of $V$ such that $\phi\left(W_{i}\right) \leq W_{i}$. If $V=\varliminf \varliminf^{*} W_{i}$, then

$$
\operatorname{ent}_{\mathbb{K}}^{\star}(V, \phi)=\sup _{i \in I} \operatorname{ent}_{\mathbb{K}}^{\star}\left(V / W_{i}, \bar{\phi}_{W_{i}}\right),
$$

where any $\bar{\phi}_{W_{i}}: V / W_{i} \rightarrow V / W_{i}$ is the continuous endomorphism induced by $\phi$.

eP5. (Change of basis) For every local basis $\mathcal{B}$ at 0 contained in $\mathcal{B}(V)$, one has ent $\mathbb{K}_{\mathbb{K}}^{\star}(V, \phi)=\sup _{U \in \mathcal{B}} H_{\mathbb{K}}^{\star}(\phi, U)$.

\section{Change of fields}

Firstly, we deal with the case of linearly compact vector spaces in order to deduce from it the general case concerning locally linearly compact spaces.

\subsection{Restriction of scalars for linearly compact vector spaces}

Let $\mathbb{F} \leq \mathbb{K}$ be a field extension of finite degree $[\mathbb{K}: \mathbb{F}] \in \mathbb{N}$. Since any $\mathbb{K}$-space can be regarded as $\mathbb{F}$-space via the inclusion $\mathbb{F} \hookrightarrow \mathbb{K}$, one has a functor of abstract vector spaces

$$
\operatorname{res}_{\mathbb{F}}^{\mathbb{K}}(-): \mathbb{K} \text { Vect } \rightarrow{ }_{\mathbb{F}} \text { Vect, }
$$


which is usually called restriction of scalars. Let $V$ be a linearly compact $\mathbb{K}$-space. By the property P11., we know that $V$ is topologically the direct product of 1-dimensional $\mathbb{K}$-spaces (endowed with the Tychonoff topology). Since the restriction functor is a right-adjoint, we get it commutes with products. Therefore, the underlying $\mathbb{F}$-space is still a direct product, i.e.,

$$
\operatorname{res}_{\mathbb{F}}^{\mathbb{K}}(V)=\prod \operatorname{res}_{\mathbb{F}}^{\mathbb{K}}(\mathbb{K}) \cong \prod \mathbb{F}^{[\mathbb{K}: \mathbb{F}]},
$$

and the topology on $V$ coincides with the product topology of $\prod \mathbb{F}^{[\mathbb{K}: \mathbb{F}]}$, i.e., $V$ can be regarded as a linearly compact $\mathbb{F}$-space. Thus it is well-defined a restriction functor

$$
\operatorname{Res}_{\mathbb{F}}^{\mathbb{K}}(-): \mathbb{K}_{\mathbb{K}} \mathrm{LC} \rightarrow{ }_{\mathbb{F}} \mathrm{LC} .
$$

for linearly compact vector spaces and field extensions. Notice that $[\mathbb{K}: \mathbb{F}]<\infty$ is a necessary condition, since $\operatorname{Res}_{\mathbb{K}}^{\mathbb{K}}(\mathbb{K})$ has to be finite-dimensional in order to be linearly compact in the discrete topology.

\subsection{Extension of scalars for linearly compact vector spaces}

Let $\mathbb{K} \leq \mathbb{L}$ be a finite field extension, i.e., $\mathbb{L}$ is a linearly compact $\mathbb{K}$-space via multiplication. For every $V, W \in$ ${ }_{\mathbb{K}} \mathrm{LC}$, we know that $V \otimes_{\mathbb{K}} W$ is dense in $V \widehat{\otimes}_{\mathbb{K}} W$ since the complete tensor product is a topological completion of the abstract tensor product. Indeed $V \widehat{\otimes}_{\mathbb{K}} W$ is topologically spanned by the set of elements $\{v \hat{\otimes} w:=\iota(v, w) \mid$ $(v, w) \in V \times W\}$ (see (2)). Therefore one can define a structure of topological $\mathbb{L}$-space on the linearly compact $\mathbb{K}$-space $\mathbb{L} \widehat{\otimes}_{\mathbb{K}} V$, by extending the natural action

$$
l \cdot\left(l^{\prime} \hat{\otimes} v\right):=\left(l l^{\prime}\right) \hat{\otimes} v, \quad \text { for all } l, l^{\prime} \in \mathbb{L}, v \in V .
$$

We denote this topological $\mathbb{L}$-space by $\operatorname{Ind}_{\mathbb{K}}^{\mathbb{L}}(V)$.

Proposition 3.1. For every linearly compact $\mathbb{K}$-space $V$ and a finite extension $\mathbb{K} \leq \mathbb{L}$, the induced $\mathbb{L}$-space $\operatorname{Ind}_{\mathbb{K}}^{\mathbb{L}}(V)$ is topologically isomorphic to

1. $\mathbb{L} \otimes_{\mathbb{K}} V$ with the topology for which a local basis at 0 are the kernels of the natural $\mathbb{L}$-maps

$$
\mathbb{L} \otimes_{\mathbb{K}} V \rightarrow \mathbb{L} \otimes_{\mathbb{K}} V_{i}, \quad i \in I,
$$

where $V=\lim _{i \in I} V_{i}$ for some family $\left\{V_{i}\right\}$ of finite-dimensional $\mathbb{K}$-spaces.

2. $\prod_{J} \mathbb{L}$ with Tychonoff topology, where $V$ has been regarded as $\prod_{J} \mathbb{K}$.

In particular, $\operatorname{Ind}_{\mathbb{K}}^{\mathbb{L}}(V)$ is a linearly compact $\mathbb{L}$-space.

Proof. Note that

$$
V \cong \text { top } \lim _{F \in \mathcal{F}(J)} \prod_{j \in F} \mathbb{K}
$$

where $\mathcal{F}(J)$ is the directed set of all finite subsets of $J$. Thus 1. follows by (3) and Proposition 2.1 since $\mathbb{L} \cong \mathbb{K}^{n}$ for some $n$. Moreover, 2. follows by 1 . .

Therefore, whenever $\mathbb{L}$ is an extension of $\mathbb{K}$ of finite degree, the functor

$$
\operatorname{Ind}_{\mathbb{K}}^{\mathbb{L}}(-):{ }_{\mathbb{K}} \mathrm{LC} \rightarrow \mathbb{L}_{\mathbb{L}} \mathrm{LC},
$$

is defined and it is left-adjoint to the restriction functor $\operatorname{Res}_{\mathbb{K}}^{\mathbb{L}}(-)$, i.e., $\mathbb{L} \widehat{\otimes}_{\mathbb{K}} V$ satisfies the following universal property: for every linearly compact $\mathbb{L}$-space $W$ and every continuous $\mathbb{K}$-linear map $f: V \rightarrow W$ there exists a 
unique continuous $\mathbb{L}$-linear map $\hat{f}: \mathbb{L} \widehat{\otimes}_{\mathbb{K}} V \rightarrow W$ such that

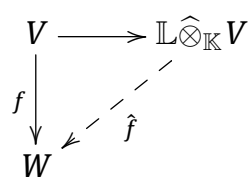

commutes. The functor $\operatorname{Ind}_{\mathbb{K}}^{\mathbb{L}}(-)$ is called induction functor.

\subsection{Restriction and Induction over ${ }_{\mathbb{K}}$ LLC}

Let $\mathbb{F} \leq \mathbb{K} \leq \mathbb{L}$ be finite field extensions. Here we define the restriction functor

$$
\operatorname{Res}_{\mathbb{F}}^{\mathbb{K}}(-): \mathbb{K}_{\mathbb{K}} L L C \rightarrow{ }_{\mathbb{F}} L L C .
$$

and the induction functor

$$
\operatorname{Ind}_{\mathbb{K}}^{\mathbb{L}}(-):{ }_{\mathbb{K}} L L C \rightarrow{ }_{\mathbb{L}} L L C,
$$

over the category of locally linearly compact vector spaces relying on the construction given above for linearly compact vector spaces. With abuse of notation, those functors shall still be denoted by Res.(-) and Ind.(-), where ${ }^{\star}, \bullet \in\{\mathbb{F}, \mathbb{K}, \mathbb{L}\}$. It unlikely causes confusion since the functors defined here coincide with (5) and (6) over the corresponding subcategory of linearly compact vector spaces.

Let $V$ be an object in ${ }_{\mathbb{K}}$ LLC. The locally linearly compact $\mathbb{K}$-space $V$ can be regarded as a locally linearly compact $\mathbb{F}$-space $\operatorname{Res}_{\mathbb{F}}^{\mathbb{K}}(V)$ since the topology on $V$ is locally generated at 0 by

$$
\left\{\operatorname{Res}_{\mathbb{F}}^{\mathbb{K}}(U) \mid U \text { linearly compact open in } V\right\},
$$

(compare with (5)). Analogously, $\operatorname{Ind}_{\mathbb{K}}^{\mathbb{L}}(V)$ is defined to be the abstract $\mathbb{L}$-space $\mathbb{L} \otimes_{\mathbb{K}} V$ together with the topology locally generated at 0 by

$$
\left\{\operatorname{Ind}_{\mathbb{K}}^{\mathbb{L}}(U) \mid U \text { linearly compact open in } V\right\},
$$

where each $\operatorname{Ind}_{\mathbb{K}}^{\mathbb{L}}(U)$ can be identified with a $\mathbb{K}$-subspace of $\mathbb{L} \otimes_{\mathbb{F}} V$ since $\mathbb{L}$ is free over $\mathbb{K}$ (compare with Proposition 3.1 and (6)). Finally, one checks that the functors so defined are additive and form the adjunction

$$
\left.\operatorname{CHom}_{\mathbb{L}}\left(\operatorname{Ind}_{\mathbb{K}}^{\mathbb{L}}(V), W\right) \cong \operatorname{CHom}_{\mathbb{K}}\left(V, \operatorname{Res}_{\mathbb{K}}^{\mathbb{L}}(W)\right), \quad \forall V \in \operatorname{ob}\left({ }_{\mathbb{K}} L L C\right) \text { and } W \in \operatorname{ob}_{\mathbb{L}} L L C\right) .
$$

\section{Topological entropy after induction and restriction}

Clearly, every functor $F:{ }_{\star}$ LLC $\rightarrow$ •LLC induces a functor of flows by $(V, \phi) \mapsto(F V, F \phi)$. Therefore, for an arbitrary discrete field $\mathbb{E}$, we simplify the notation of $\S 2.2$ as following:

N1. $C_{n}^{\bullet}(F(\phi, U)):=C_{n}^{\bullet}(F(\phi), F(U))$, for all $n \in \mathbb{N}$;

N2. $H_{\mathbb{E}}^{\star}(F(\phi, U)):=H_{\mathbb{E}}^{\star}(F(\phi), F(U))$;

N3. $\operatorname{ent}_{\mathbb{E}}^{\star}(F(V, \phi)):=\operatorname{ent}_{\mathbb{E}}^{\star}(F(V), F(\phi))$.

\subsection{Linearly compact case}

Let $\mathbb{F} \leq \mathbb{K} \leq \mathbb{L}$ be field extensions such that $[\mathbb{L}: \mathbb{F}]<\infty$. Let $V$ be a linearly compact vector $\mathbb{K}$-space and let

$$
{ }_{v} \beta: \prod_{n=0}^{\infty} V \rightarrow \prod_{n=0}^{\infty} V, \quad\left(v_{n}\right)_{n \in \mathbb{N}} \mapsto\left(v_{n+1}\right)_{n \in \mathbb{N}},
$$


denote the left Bernoulli shift of $V$. For $V=\mathbb{K}$, the map $\mathbb{K} \beta$ is the one-dimensional left Bernoulli shift whose topological entropy is 1 (see [6, Example 3.16(a)]). Applying the restriction functor Res $\mathbb{K}_{\mathbb{K}}^{\mathbb{K}}$ to the flow $\left(\prod_{n=0}^{\infty} \mathbb{K}, \mathbb{K} \beta\right)$ produces an endomorphism of $\operatorname{Res}_{\mathbb{F}}^{\mathbb{K}}\left(\prod_{n=0}^{\infty} \mathbb{K}\right)=\prod_{n=0}^{\infty} \mathbb{F}^{[\mathbb{K}: \mathbb{F}]}$. In particular, it coincides to the left Bernoulli shift $t_{\mathbb{F}[\mathbb{K}: \mathbb{F}]} \beta$ of the $\mathbb{F}$-space $\mathbb{F}^{[\mathbb{K}: \mathbb{F}]}$ whose topological entropy equals $[\mathbb{K}: \mathbb{F}]$ by $[6$, Example 3.16(b)]. Therefore,

$$
\operatorname{ent}_{\mathbb{F}}^{\star}\left(\operatorname{Res}_{\mathbb{F}}^{\mathbb{K}}\left(\prod_{n=0}^{\infty} \mathbb{K}, \mathbb{K} \beta\right)\right)=\operatorname{ent}_{\mathbb{F}}^{\star}\left(\prod_{n=0}^{\infty} \mathbb{F}^{[\mathbb{K}: \mathbb{F}]},{ }_{\mathbb{F}[\mathbb{K}: \mathbb{P}} \beta\right)=[\mathbb{K}: \mathbb{F}]=[\mathbb{K}: \mathbb{F}] \cdot \operatorname{ent}_{\mathbb{K}}^{\star}\left(\prod_{n=0}^{\infty} \mathbb{K}, \mathbb{K} \beta\right) .
$$

On the other hand, the induction $\operatorname{Ind}_{\mathbb{K}}^{\mathbb{L}}$ produces an endomorphism of the $\mathbb{L}$-space $\prod_{n=0}^{\infty} \mathbb{L}$ that coincides to the (1-dimensional) left Bernoulli shift $\mathbb{L} \beta$ of $\mathbb{L}$. In particular,

$$
\operatorname{ent}_{\mathbb{L}}^{\star}\left(\operatorname{Ind}_{\mathbb{K}}^{\mathbb{L}}\left(\prod_{n=0}^{\infty} \mathbb{K}, \mathbb{K} \beta\right)\right)=\operatorname{ent}_{\mathbb{L}}^{\star}\left(\prod_{n=0}^{\infty} \mathbb{L}, \mathbb{L} \beta\right)=1=\operatorname{ent}_{\mathbb{K}}^{\star}\left(\prod_{n=0}^{\infty} \mathbb{K}, \mathbb{K} \beta\right) .
$$

The following result shows that the formulae above hold for arbitrary flows over $\mathbb{K}_{\mathbb{K}} \mathrm{LC}$.

Proposition 4.1. Let $\mathbb{F} \leq \mathbb{K} \leq \mathbb{L}$ such that $[\mathbb{L}: \mathbb{F}]<\infty$. For every flow $(V, \phi)$ over $\mathbb{K}_{\mathbb{K}} \mathrm{LC}$ one has

$$
\operatorname{ent}_{\mathbb{F}}^{\star}\left(\operatorname{Res}_{\mathbb{F}}^{\mathbb{K}}(V, \phi)\right)=[\mathbb{K}: \mathbb{F}] \cdot \operatorname{ent}_{\mathbb{K}}^{\star}(V, \phi) \text { and } \operatorname{ent}_{\mathbb{L}}^{\star}\left(\operatorname{Ind}_{\mathbb{K}}^{\mathbb{L}}(V, \phi)\right)=\operatorname{ent}_{\mathbb{K}}^{\star}(V, \phi) \text {. }
$$

For a field $\mathbb{E}$, the collection of all open $\mathbb{E}$-subspaces $\prod A_{i}$ of $\prod \mathbb{E}$ such that

(G1) $A_{i}=\mathbb{E}$ for all but finitely many indices,

(G2) $A_{i}=0$ otherwise;

form a neighbourhood basis at 0 for the Tychonoff topology on $\prod \mathbb{E}$. We will refer to such a basis as good basis of $\prod \mathbb{E}$ and its elements will be said to be good.

Proof of Proposition 4.1. For an arbitrary flow $(V, \phi)$ over ${ }_{\mathbb{K}} \mathrm{LC}$, let $U=\prod A_{i}$ be a good $\mathbb{K}$-subspace of $V$. Thus

$$
\begin{aligned}
H_{\mathbb{F}}^{\star}\left(\operatorname{Res}_{\mathbb{F}}^{\mathbb{K}}(\phi, U)\right) & =\lim _{n \rightarrow \infty} \frac{1}{n} \cdot \operatorname{codim}_{\mathbb{F}} C_{n}^{\mathbb{F}}\left(\operatorname{Res}_{\mathbb{F}}^{\mathbb{K}}(\phi, U)\right)= \\
& =\lim _{n \rightarrow \infty} \frac{[\mathbb{K}: \mathbb{F}]}{n} \cdot \operatorname{codim}_{\mathbb{K}} C_{n}^{\mathbb{K}}(\phi, U)=[\mathbb{K}: \mathbb{F}] \cdot H_{\mathbb{K}}^{\star}(\phi, U) .
\end{aligned}
$$

and notice that $\operatorname{Res}_{\mathbb{F}}^{\mathbb{K}}(U)$ is a good $\mathbb{F}$-subspace of $V$. Since good subspaces form a neighbourhood basis at 0 , one has that

$$
[\mathbb{K}: \mathbb{F}] \cdot \operatorname{ent}_{\mathbb{K}}^{\star}(V, \phi) \leq \operatorname{ent}_{\mathbb{F}}^{\star}\left(\operatorname{Res}_{\mathbb{F}}^{\mathbb{K}}(V, \phi)\right) .
$$

Finally, the equality holds since every good $\mathbb{F}$-subspace of $\operatorname{Res}_{\mathbb{F}}^{\mathbb{K}}(V)$ can be realised as the restriction of a good $\mathbb{K}$-subspace of $V$. In order to prove the second part of the statement, notice that

$$
\operatorname{dim}_{\mathbb{K}}(-)=\operatorname{dim}_{\mathbb{L}}\left(\operatorname{Ind}_{\mathbb{K}}^{\mathbb{L}}(-)\right) \quad \text { and } \quad C_{n}^{\mathbb{L}}\left(\operatorname{Ind}_{\mathbb{K}}^{\mathbb{L}}(\phi, U)\right)=\operatorname{Ind}_{\mathbb{K}}^{\mathbb{L}}\left(C_{n}^{\mathbb{K}}(\phi, U)\right),(U \in \mathcal{B}(V), n \in \mathbb{N}) .
$$

Therefore,

$$
\begin{aligned}
H_{\mathbb{L}}^{\star}\left(\operatorname{Ind}_{\mathbb{K}}^{\mathbb{L}}(\phi, U)\right) & =\lim _{n \rightarrow \infty} \frac{1}{n} \cdot \operatorname{codim}_{\mathbb{L}} C_{n}^{\mathbb{L}}\left(\operatorname{Ind}_{\mathbb{K}}^{\mathbb{L}}(\phi, U)\right)=\lim _{n \rightarrow \infty} \frac{1}{n} \cdot \operatorname{codim}_{\mathbb{L}} \operatorname{Ind}_{\mathbb{K}}^{\mathbb{L}}\left(C_{n}(\phi, U)\right)= \\
& =\lim _{n \rightarrow \infty} \frac{1}{n} \cdot \operatorname{codim}_{\mathbb{K}} C_{n}(\phi, U)=H_{\mathbb{K}}^{\star}(\phi, U) .
\end{aligned}
$$

Same reasoning as above yields ent $t_{\mathbb{L}}^{\star}\left(\operatorname{ind}_{\mathbb{K}}^{\mathbb{L}}(V, \phi)\right)=\operatorname{ent}_{\mathbb{K}}^{\star}(V, \phi)$.

Remark 4.2. An easy observation yields that, for every linearly compact $\mathbb{K}$-space $V$, one has

$$
\operatorname{ent}_{\mathbb{L}}^{\star}\left(\operatorname{Ind}_{\mathbb{F}}^{\mathbb{L}}\left(\operatorname{Res}_{\mathbb{F}}^{\mathbb{K}}(V, \phi)\right)\right)=[\mathbb{K}: \mathbb{F}] \cdot \operatorname{ent}_{\mathbb{K}}^{\star}(V, \phi) \quad \text { and } \quad \operatorname{ent}_{\mathbb{F}}^{\star}\left(\operatorname{Res}_{\mathbb{F}}^{\mathbb{L}}\left(\operatorname{Ind}_{\mathbb{K}}^{\mathbb{L}}(V, \phi)\right)\right)=[\mathbb{L}: \mathbb{F}] \cdot \operatorname{ent}_{\mathbb{K}}^{\star}(V, \phi) \text {. }
$$

Using induction and restriction gives back an intuitive method to generate examples of topological flows with some finite topological entropy $n \in \mathbb{N}$ over different fields. E.g., let $(V, \mathbb{K} \beta)$ be the 1-dimensional left Bernoulli shift defined above. One easily generates the $n$-dimensional left Bernoulli shift over $\mathbb{K}$ by combining induction and restriction with respect to a field extension of $\mathbb{K}$ of degree $n$. 
Remark 4.3. In [4] a deep relationship between a flow $(V, \phi)$ and the Bernoulli shift has been recently proved: the flow $(V, \phi)$ is essentially a product of one-dimensional left Bernoulli shifts as many as ent $\mathbb{K}_{\mathbb{K}}^{\star}(V, \phi)$ counts. Therefore, Proposition 4.1 can be obtained as corollary of [4, Theorem A].

\subsection{General case}

Recall that every locally linearly compact $\mathbb{K}$-space $V$ can be split into a topological sum of a linearly compact $\mathbb{K}$-space $V_{c}$ and a discrete $\mathbb{K}$-space $V_{d}$; namely, $V \cong{ }_{t o p} V_{c} \oplus V_{d}$. Actually, this type of decomposition is available whenever we consider a linearly compact open subspace $U$ of $V$. Indeed, $V \cong{ }_{t o p} U \oplus V / U$.

Now let $(V, \phi)$ be a flow over $\mathbb{K}_{\mathbb{K}} \mathrm{LLC}$. Any decomposition $V \cong_{t o p} V_{c} \oplus V_{d}$ induces the decomposition

$$
\phi=\left(\begin{array}{ll}
\phi_{c c} & \phi_{d c} \\
\phi_{c d} & \phi_{d d}
\end{array}\right)
$$

where $\phi_{\bullet} * V_{\bullet} \rightarrow V_{\star}$ is the composition $\phi_{\bullet} \star p_{\star} \circ \phi \circ l_{\bullet}$ for $\bullet,{ }^{\star} \in\{c, d\}$. Therefore, $\phi_{\bullet} \star$ is continuous being composition of continuous homomorphisms.

Lemma 4.4. Let $\mathbb{F} \leq \mathbb{K} \leq \mathbb{L}$ be finite field extensions and $V \cong{ }_{\text {top }} V_{c} \oplus V_{d}$ a locally linearly compact $\mathbb{K}$-space together with a continuous endomorphism $\phi: V \rightarrow V$. Thus

1. $\operatorname{Res}_{\mathbb{F}}^{\mathbb{K}}(V)$ admits a decomposition such that

$$
\operatorname{Res}_{\mathbb{F}}^{\mathbb{K}}(V)_{c}=\operatorname{Res}_{\mathbb{F}}^{\mathbb{K}}\left(V_{c}\right) \quad \text { and } \quad \operatorname{Res}_{\mathbb{F}}^{\mathbb{K}}(\phi)_{c c}=\operatorname{Res}_{\mathbb{F}}^{\mathbb{K}}\left(\phi_{c c}\right) .
$$

2. $\operatorname{Ind}_{\mathbb{K}}^{\mathbb{L}}(V)$ admits a decomposition such that

$$
\operatorname{Ind}_{\mathbb{K}}^{\mathbb{L}}(V)_{c}=\operatorname{Ind}_{\mathbb{K}}^{\mathbb{L}}\left(V_{c}\right) \quad \text { and } \quad \operatorname{Ind}_{\mathbb{K}}^{\mathbb{L}}(\phi)_{c c}=\operatorname{Ind}_{\mathbb{F}}^{\mathbb{L}}\left(\phi_{c c}\right) .
$$

Proof. Notice that $\operatorname{Re}_{\mathbb{F}}^{\mathbb{K}}(V)$ (or $\operatorname{Ind}_{\mathbb{K}}^{\mathbb{L}}(V)$ ) can be obtained by restricting (or extending) $V_{c}$ and $V_{d}$ first in the corresponding categories and then summing them up in $\mathbb{K}_{\mathbb{K}} L L C$. Indeed both restriction functor and induction functor are additive.

Theorem 4.5. Let $\mathbb{F} \leq \mathbb{K} \leq \mathbb{L}$ such that $[\mathbb{L}: \mathbb{F}]<\infty$. For every flow $(V, \phi)$ over $\mathbb{K} L L C$ one has

$$
\operatorname{ent}_{\mathbb{F}}^{\star}\left(\operatorname{Res}_{\mathbb{F}}^{\mathbb{K}}(V, \phi)\right)=[\mathbb{K}: \mathbb{F}] \cdot \operatorname{ent}_{\mathbb{K}}^{\star}(V, \phi) \quad \text { and } \quad \operatorname{ent}_{\mathbb{L}}^{\star}\left(\operatorname{Ind}_{\mathbb{K}}^{\mathbb{L}}(V, \phi)\right)=\operatorname{ent}_{\mathbb{K}}^{\star}(V, \phi) \text {. }
$$

Proof. By the previous lemma, it follows directly from Proposition 4.1 and the reduction to linearly compact vector spaces devoloped in [6, Section 4].

Acknowledgement: This work was supported by EPSRC Grant N007328/1 Soluble Groups and Cohomology and partially supported by Programma SIR 2014 del MIUR, Progetto GADYGR, Numero RBSI14V2LI, cup G22I15000160008.

\section{References}

[1] R. L. Adler, A. G. Konheim and M. H. McAndrew, Topological entropy. Trans. Amer. Math. Soc., 114, (1965): 309-319.

[2] F. Berlai, D. Dikranjan and A. Giordano Bruno, Scale function vs Topological entropy. Topology and its Applications, 160(18), (2013): 2314-2334.

[3] R. Bowen, Entropy for group endomorphisms and homogeneous spaces. Trans. Amer. Math. Soc., 153, (1971): 401-414.

[4] I. Castellano. The corank of a flow over the category of linearly compact vector spaces. Journal of Pure and Applied Algebra, 224(6), (2020): 106-266 
[5] I. Castellano and A. Giordano Bruno, Algebraic entropy in locally linearly compact vector spaces. Rings, Polynomials, and Modules. Springer, Cham, (2017): 103-127.

[6] I. Castellano and A. Giordano Bruno, Topological entropy for locally linearly compact spaces. Topology and its Application, 252, (2019): 112-144.

[7] B.M. Hood, Topological entropy and uniform spaces J. London Math. Soc., 8(2), (1974): 633-641.

[8] A. Giordano Bruno and S. Virili, Topological entropy in totally disconnected locally compact groups. Ergodic Theory and Dynamical Systems, 37(7), (2017): 2163-2186.

[9] L. Ribes and P. Zalesskii, Profinite groups. Springer, Berlin, Heidelberg, (2000).

[10] G.A. Willis, The structure of totally disconnected locally compact groups. Math. Ann., (1994): 341-363. 